\title{
Assessment of Student Learning Experience in Two Exemplary Engineering Projects
}

\section{Dr. Wookwon Lee, Gannon University}

Wookwon Lee, P.E. received the B.S. degree in electronic engineering from Inha University, Korea, in 1985, and the M.S. and D.Sc. degrees in electrical engineering from the George Washington University, Washington, DC, in 1992 and 1995, respectively. He is currently an associate professor and department chair of the Department of Electrical and Computer Engineering at Gannon University, Erie, PA. Prior to joining Gannon, he had been involved in various research and development projects in industry and academia for more than 15 years.

\section{Dr. Nicholas B. Conklin, Gannon University}

Nicholas B. Conklin received a B.S. in applied physics from Grove City College in 2001, and a Ph.D. in physics from Penn State University in 2009. He is currently an associate professor and chair of the Physics Department at Gannon University, Erie, PA. 


\title{
Assessment of Student Learning Experience in Two Exemplary Engineering Projects
}

\begin{abstract}
In this paper, we examine and quantify similarities of two engineering projects each of which is either 1) an undergraduate research project primarily integrating off-the-shelf devices and referred to as the "system integration and configuration project" or 2) a project primarily involving bottomup implementation using discrete electronic components and ICs while conceived in a top-down design and referred to as the "design and implementation project." For the quantification of similarities and characteristics of project activities, we apply the Cohen's kappa to survey data obtained from a group of students who have been on both types of projects. Our analysis indicates that the quantification technique using the Cohen's kappa could be a useful tool to evaluate similarities of two engineering projects in addressing student learning outcomes.
\end{abstract}

\section{Introduction}

Over the past seven years, we have carried out a series of extracurricular engineering projects to engage undergraduate students in research with a goal of enhancing the student learning experience and contributing to further improving student learning outcomes, especially for electrical and computer engineering students. Under a small-scale umbrella program called High-Altitude Ballooning (HAB), these projects have been financially supported in part by external sponsors, mostly NASA or its Space Grant Consortia, and administered in the form of extracurricular activities. Recent assessments about our program outcomes and student learning outcomes showed that our program has been fairly successful in achieving its goals and objectives [1][2].

Since fall 2015 and in the 2016-2017 academic year, we have been running two projects concurrently with the same group of students working on both projects. As we have carried out these projects, we observed that student team members seem to have developed a preference for one project over the other. Although both projects were generally perceived as very interesting and challenging, one project involved more integration and configuration of "off-the-shelf" devices, such as Wi-Fi modems, radio frequency (RF) communication devices, microcontrollers, and cameras, as well as operating the complete system, while the other project involved more actual design of electronic devices from discrete electrical/electronic components and integrated circuits (ICs) as well as soldering and constructing physical hardware structures to build subsystems into an entire system.

In this paper, we examine and quantify these two projects for comparison purposes in the context of 1) a project integrating off-the-shelf devices (referred to as the "system integration and configuration project") and 2) a project including bottom-up implementation using discrete components and ICs to build a complete system conceived in a top-down design approach (referred to as the "design and implementation project"). Detailed descriptions for both projects are provided to allow for an accurate comparison. Following our characterization of the two example engineering projects, we present survey data and a quantitative analysis on student perceptions to 
examine any difference in achieving student learning outcomes. Understanding which types of projects most successfully enhance student learning outcomes will aid in designing future projects to attract, retain, and effectively educate students.

\section{Example Project 1: "Integration and Configuration Project"}

\subsection{Subsystem Description - Solar Eclipse}

The integration and configuration project involves configuring a ballooning system consisting of a ground station and several high-altitude balloon payloads to live-stream high definition video of the August 21, 2017 solar eclipse. This project is part of a nation-wide effort, spearheaded by NASA and the national project

office at the Montana State University (MSU), to capture video of the entire eclipse path across the United States. A baseline system was provided to all solar eclipse participants by the national project office. A functional block diagram of the balloon system is show in Figure 1 which is largely comprised of a balloon system, a baseline ground station, and an MTS mobile station. The balloon system consists of five payloads which include four baseline payloads for still image, video, Iridium, and cut-down, of the baseline system. The fifth payload, MTS-Tx is an additional payload for a 3-mode tracking subsystem under test intended to establish reliable tracking capabilities at the University without having to rely on any existing satellite systems.

In the baseline system [3], video is captured using a Raspberry Pi and camera, transmitted using a $5 \mathrm{GHz}$ RF modem in the balloon system, and received by a second RF modem in the baseline ground station which also includes a dish antenna and a laptop computer. A still image capture system, operating at $900 \mathrm{MHz}$, functions similarly as a backup. Position and tracking are accomplished using an Iridium satellite modem and GPS position reporting server operated by the national project office. The additional payload for the multiband tracking system (MTS) integrates an RF modem, amateur radio's automatic packet reporting system (APRS) transmitter, and a cell phone in the payload and their receiving components in the MTS mobile station (for more details, refer to [4]). For the project activities, since the baseline system was provided to all participating teams, the main, but not insignificant, challenge is learning to configure and operate it efficiently and successfully to capture images and streaming video of the solar eclipse on August 21, 2017. 


\subsection{Description of Project Activities}

In order to properly configure and operate the baseline balloon system, key learning took place on the following aspects [4]:

- Configuring the Raspberry Pi: as all engineering team members are on the electrical engineering track as opposed to computer engineering track, configuring the Raspberry Pi via a remote SSH login to the Linux environment required a significant amount of learning which included learning basic commands and recognizing major differences between Windows-based and Linux-based operating systems, as well as learning basics of computer networking to properly configure the GUI (e.g., communication ports) on the laptop for the modems on the ground station.

- Reading and learning from the datasheets and manuals about the modems used, i.e., RFD900+ at $900 \mathrm{MHz}, \mathrm{M} 5$ at $5.8 \mathrm{GHz}$ and Iridium, as none of the team members had basic knowledge in communications from their coursework yet.

- Compiling the team's own configuration and operating procedure to test each and every functional subsystem in the baseline payloads from the individual payload manuals provided by the national project office.

In addition, as noted above, the MTS payload focuses largely on the integration of commercial offthe-shelf (COTS) components rather than designing custom electronics. This payload makes use of three different tracking technologies, RF, APRS, and cellphone, to aid in the recovery of the balloon system. All three tracking subsystems are integrated with a single microcontroller and GPS module. Below we further describe each subsystem and the student learning that is required to complete the subsystem.

For the RF subsystem, students programmed a microcontroller to read and parse the National Marine Electronics Association (NMEA) formatted data strings from a GPS module. They then learned to transmit the payload position to the mobile ground station using a serial connection via RF modem. The MTS mobile station was also equipped with an RF modem, and students learned to configure the devices to correctly communicate with one another. When the RF modem of the MTS mobile station receives the custom formatted position coordinates, they are input to a custombuilt $\mathrm{C} \#$ code to plot the position coordinates on our Google Earth-based mapping software. The majority of this code already existed from another project, but students adapted it to read the position string directly from the serial connection.

Line-of-sight (LOS) communication must be maintained with the payload for the MTS to function correctly. Therefore, students must use existing balloon trajectory software to forecast the projected flight path so that the MTS mobile station can be deployed somewhere between the launch site and the expected landing site, since the balloon can travel in excess of $100 \mathrm{mph}$ in the stratosphere and a chase vehicle cannot (legally) keep up.

The $144 \mathrm{MHz}$ amateur radio band is reserved for the APRS system, which can transmit GPS coordinates. The APRS subsystem functions by sending GPS strings from the microcontroller to an APRS encoder connected to a radio. The radio signal is then received by an APRS receiver on the MTS mobile station, which is plotted similarly to the RF subsystem. The APRS subsystem 
does not require LOS communication with the payload, but in theory must be within radio range. In practice, APRS packets are typically repeated by local "digipeaters" independently operated by amateur radio enthusiasts, and the tracking information can thus travel hundreds of miles. In order

to implement the APRS subsystem, students selected a commercially available APRS encoder and radio as well as VHF antennas from the many options available. They learned how to configure the encoder using a PC, integrate a GPS module with the encoder, and operate the radio. In order to use the amateur radio, students also had to study for and pass the amateur radio "technician" class licensing exam. Students must also learn to operate the APRS receiver and integrate it with a $\mathrm{PC}$ to extract the position coordinates.

Finally, for the cellphone-based tracking, the microcontroller in the payload will monitor the GPS coordinates, which should continue to change until balloon comes to rest. When this condition is met, a power relay will be used to turn on a cell phone running tracking software. The phone is turned on while on the ground, rather than being left on during flight, because FCC regulations prevent the operation of a cell phone radios during balloon or airplane flights. Once the payload comes to rest, a website operated by the tracking app provider will display the position of the payload. Implementation of the cellphone tracker will require students to program the microcontroller to monitor GPS coordinates and set switch-on/off conditions upon which to activate the cellphone. They are also learning to use prebuilt software in the Android ecosystem.

\section{Example Project 2: "Design and Implementation Project"}

\subsection{Subsystem Description - CHERP}

As part of NASA's Undergraduate Student Instrumentation Project (USIP), the authors are mentoring a multi-disciplinary team of undergraduate students to design, build, test, and fly a custom-designed balloon-born cosmic-ray detector called the CHErenkov Radiator Payload (CHERP). Cosmic rays are high-energy particles, predominantly protons, but including electrons and heavier nuclei as well, arriving at Earth from outside the solar system. The payload is designed to measure the cosmic-ray proton-to-helium ratio as a function of energy from $\sim 1.5$ to $20 \mathrm{GeV} /$ nucleon, which can provide insight into the production and propagation history of cosmic rays. A balloon will carry the payload to an altitude of $\sim 36 \mathrm{~km}$ to sample cosmic rays before they interact in the atmosphere. A top-level functional block diagram of the payload is shown in Figure 2.

The data collection module shown in Figure 2 is composed of two Cherenkov detectors and a charge detector. Cherenkov radiation occurs when a particle travels through a material faster than light travels through that material, $v>c / n$, where $v$ is particle speed, $c$ is the speed of light, and $n$ is the material's index of refraction. The amount of Cherenkov light depends on the particle speed, with particles traveling closer to the speed of light emitting more light until saturation occurs. Cherenkov detectors are "blind" to particles below this threshold speed. In order to extend the energy range and provide cross-calibration, two Cherenkov radiators with different indices of refraction will be used in the payload. Cherenkov light will be measured by sensitive light sensors known as silicon photomultipliers (SiPMs). Each Cherenkov radiator is readout by four SiPMs. 


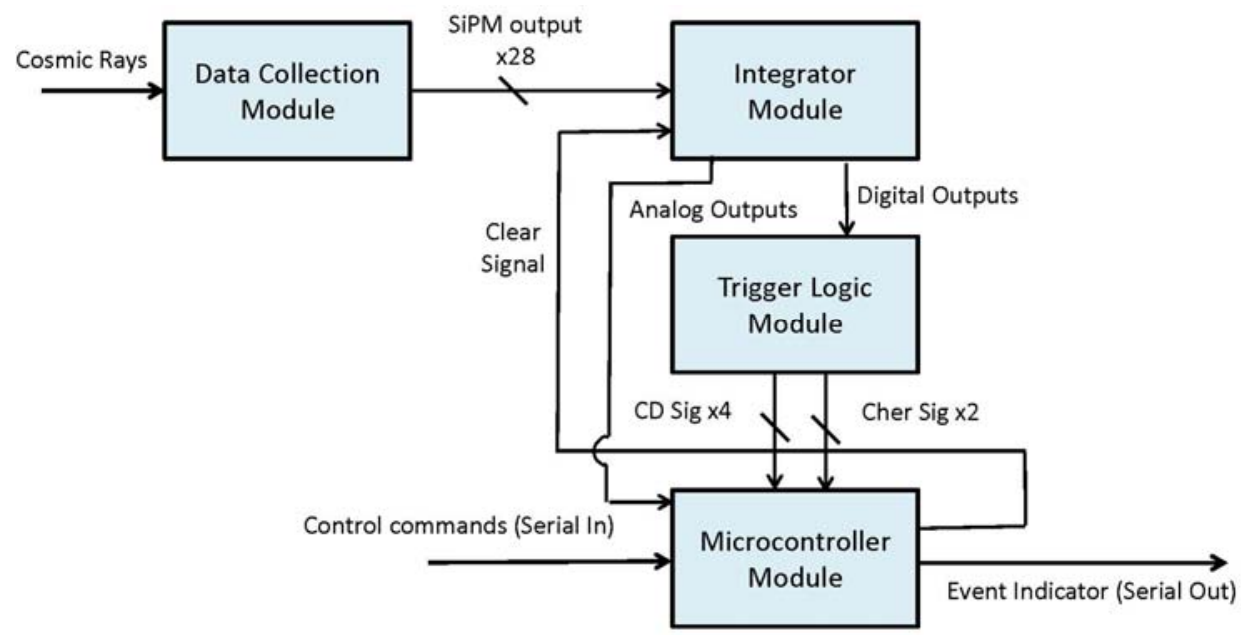

Figure 2. CHERP functional block diagram

Distinguishing protons from helium (and heavier) nuclei can be accomplished by measuring their charge, since protons have a charge of $Z=1$ and helium nuclei have a charge of $Z=2$, in units of elemental charge. Charge detection will be accomplished using scintillator, which is a special type of material that emits light, proportional to $\mathrm{Z}^{2}$, when traversed by charged particles. Scintillator light will also be read out using SiPMs. In order to eliminate stray light from the environment, scintillators are wrapped in black electrical tape. There will be four scintillating layers, two at the top of the instrument and two at the bottom. Each layer consists of five scintillator paddles, which are arranged in crossed layers to provide rudimentary tracking information.

As shown in Figure 3, SiPM output is input to a custom student-designed integrator module circuit. Since the output current of the SiPMs is proportional to the amount of light incident on them, the

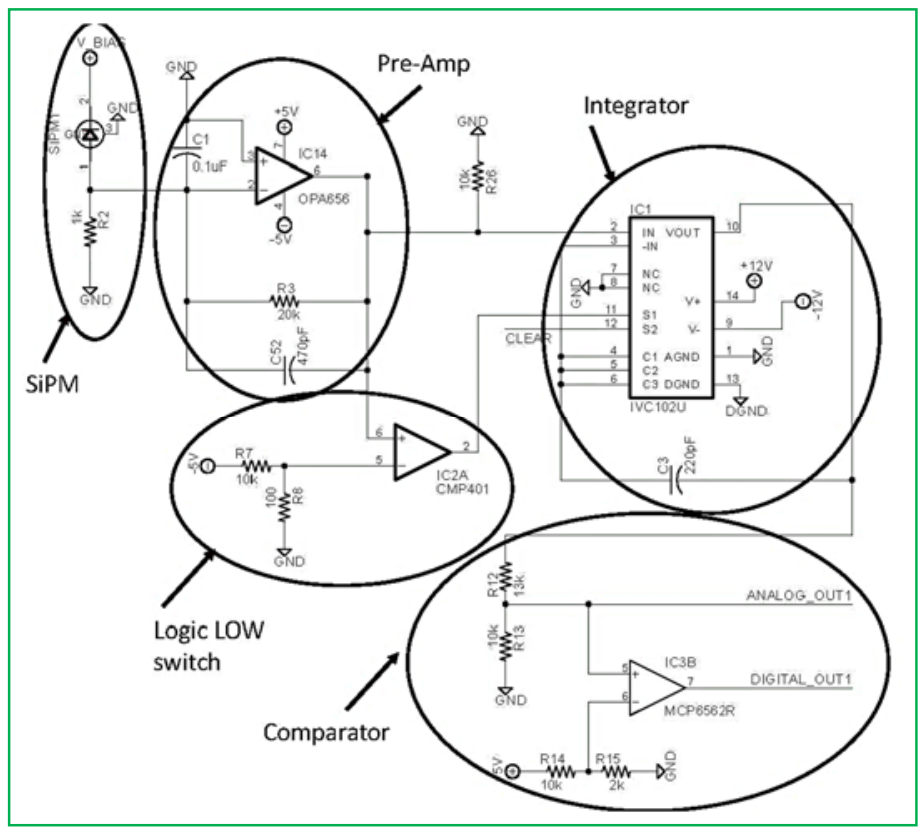

Figure 3. Pre-amplifier and integrator circuits for the Data Collection Module 
first stage of the circuits uses a trans-impedance amplifier to amplify and convert the SiPM output current to a voltage. The next stage of the circuit employs a Burr-Brown IVC102 precision switched integrator. When the output voltage of the integrator exceeds a small reference voltage $(\sim 50 \mathrm{mV})$, a comparator output switches to TTL high to indicate that the SiPM has data, and the analog output voltage is held until it can be readout by an ADC. Each SiPM is connected to a separate integrator circuit, and the circuit has been implemented such that five integrators are included on a single printed circuit board (PCB). This allows a single PCB to service an entire scintillator or Cherenkov layer.

The trigger logic module is composed of a Mojo v3 FPGA with a custom-designed interface board. The digital output of each integrator module channel is input to the trigger logic module for a total of 28 inputs. The trigger logic module has six outputs, one for each scintillator and Cherenkov layer. The trigger logic output is constructed by logical OR operations with the five inputs of each scintillator layer and the four inputs of each Cherenkov layer. These outputs are then input to the microcontroller module and evaluated to determine if there was a good event with data in all four scintillators and at least one Cherenkov layer. For a good event, the microcontroller module initiates ADC readout of the analog integrator outputs, while for a bad event, a digital CLEAR signal is sent to the integrator module to clear the integrator outputs and prepare for the next event.

The microcontroller module is constructed of an Arduino Due microcontroller with a custom frontend board including a real-time clock, SD card reader, and two AD7490 16-channel 12-bit ADCs. For a good event, each integrator analog output voltage is readout by an ADC and written to the SD card for later analysis. The microcontroller also monitors time, two temperature sensors, and handles serial communication with the flight provider.

A key difference between the solar eclipse and CHERP projects, especially for the balloon flight, is how the payloads are launched. For the solar eclipse project, students are responsible for all aspects of the balloon launch, including flight prediction; laying out the payload, parachute, and balloon for launch; inflating the balloon to provide the proper lift; monitoring the position of the payload in real time; and recovery. On the other hand, the CHERP project relies entirely on a $3^{\text {rd }}$ party flight provider to handle all aspects of the logistics. The goal is simply to design the science payload.

\subsection{Description of Project Activities}

Project activities on the payload design and implementation began in January 2016 for preliminary tasks within the student team. With an official begin date in Sept 2016, design and construction of the payload are still underway, with projected completion in Spring 2018. This project has and will continue to provide a myriad of opportunities for students to learn about designing and building a system from the ground up.

Despite the different physics responsible for generating the light, both components of the data collection module, the Cherenkov and charge detectors, use identical readout electronics. Students are responsible for all aspects of designing the readout electronics, including selecting the SiPM vendor and model, designing the SiPM biasing electronics, testing the SiPMs, designing the integrator circuit, and implementing the SiPM bias and integrator designs on PCBs. Students are 
also responsible for soldering surface mount components onto these PCBs, designing and implementing a test-plan, and debugging circuits. For the schematic circuits shown in Figure 3, the students construct the circuits on the breadboard, and verify the functionality of each circuit and the overall operation of all circuits together. Its breadboard implementation is shown in Figure 4. The construction of the circuit is relatively easy and straightforward as students have done similar work in various lab courses in the curriculum such as circuits and electronics. However, students are introduced to a new concept, the test plan, when it comes to test and verification. A test plan is a comprehensive collection of test cases that covers DC biasing of the circuits as well as AC operation of the circuits in cascade. The concept of a test plan is

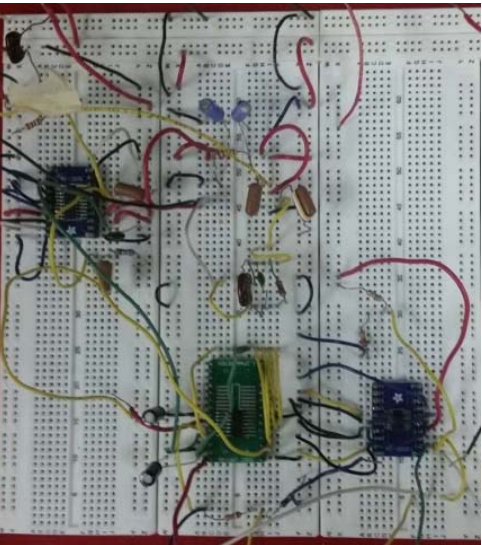

Figure 4. Breadboard implementation for verification and troubleshooting of preamplifier and integrator typically taught in the senior design course as part of system verification. Before learning of the test plan, students tend to quickly, and often prematurely, judge the operation of the circuit solely via visual verification of the ultimate output signal. Troubleshooting is inevitable almost in all breadboard implementation and lots of hands-on experience is acquired during the testing and verification stage, which may take weeks of time as opposed to relatively short, one or two class period time in a lab course.

Once the intended circuit operation was fully verified on the breadboard, it was implemented in a compact PCB design. This PCB implementation includes 1) PCB layout design shown in Figure 5(a), 2) Outsourcing of PCB fabrication and acquisition of discrete components, 3) soldering of all discrete components, ICs, and headers on the PCB as shown in Figure 5(b). Stacking up multiple PCBs and wiring them together will follow once all PCB implementation is verified and debugged if necessary. Successfully completing this stage of work takes months for a student team of 8 members. Layout design itself went through multiple reviews and revisions over a month in summer 2016, and the first PCB implementation, testing, and troubleshooting took nearly 3 months

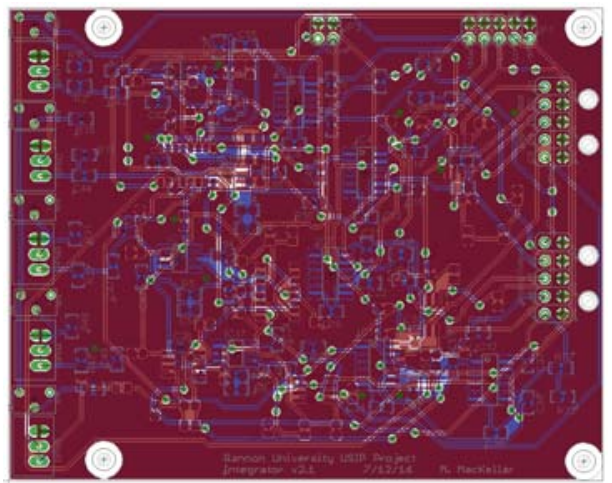

(a)

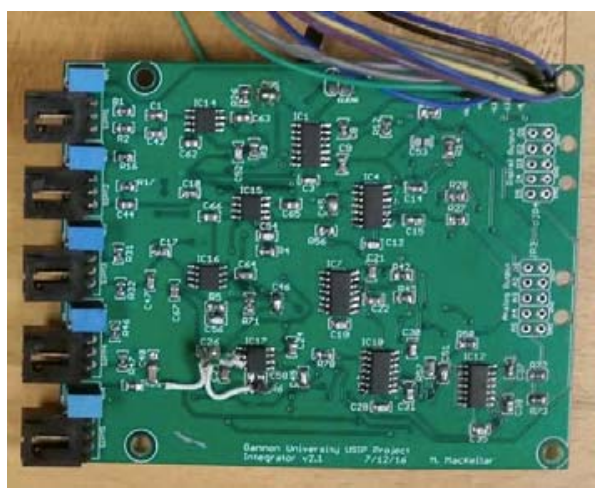

(b)

Figure 5. Printed circuit board (PCB): (a) Software-based layout design (b) PCB with discrete components soldered 
during the 2016 fall semester. In this stage of work, students do all levels of work, from low-level "dirty" work, such as soldering and cable making, to very intelligent engineering work, like debugging. Under close and proper supervision by the faculty members, students seem to truly enjoy the work and are eager to learn and enhance their skills since they do not normally get this type of comprehensive experience from lab courses in a curriculum.

A similar procedure was followed for the trigger logic module and microcontroller module. For the trigger logic board, students programmed the FPGA using VHDL, designed the interface PCB board, created and implemented a test plan, and soldered components onto the board. For the microcontroller module, the microcontroller was programmed using the Arduino IDE to read out the ADCs, handle the digital I/O in an efficient manner, write all events to an SD card, monitor temperature, and handle serial communication with the flight provider. A custom interface board was designed to incorporate all these functions into a single PCB. Figure 6(a) shows a breadboard implementation of microcontroller subsystems that will interact with the microcontroller, and Figure 6(b) is two PCBs, one for integration of the subsystems and the other as the breakout board to effectively interface with the microcontroller. The interface board also incorporates a TTL-toRS-232 line-driver/receiver to convert the TTL logic levels from the microcontroller into the RS232 logic level serial input/output used by our proposed flight provider. Also shown in Figure 6(b) is a set of cables made in house by the students as part of the "dirty" work, which includes cutting the wires to length, properly stripping off both ends of the wires, sorting and grouping by colors, wrapping with shrink tubing, and attaching connectors to both ends.

In addition to designing, building, and testing the various electronics of the payload subsystems, students are also responsible for the mechanical aspects of the payload, including gluing and wrapping of SiPMs and scintillators, designing and 3D printing the support structures, and mechanical assembly of the detector module. Once completed, the payload subsystems need to be integrated into a single functioning payload. To accomplish this, students are responsible for building and connecting the cables, testing for proper subsystem operation, and debugging problems that arise as the payloads interact with one another. For illustration purposes, Figure 7 shows an example of a similar payload built in the past. Upon completion of the payload, students, in addition to a faculty advisor, will travel to the flight provider to mechanically and electrically integrate their payload with the flight provider's system.

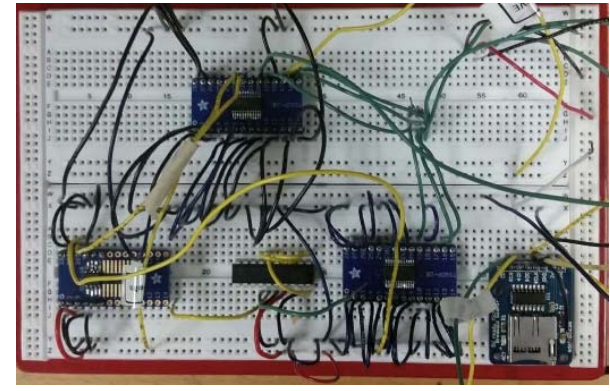

(b)

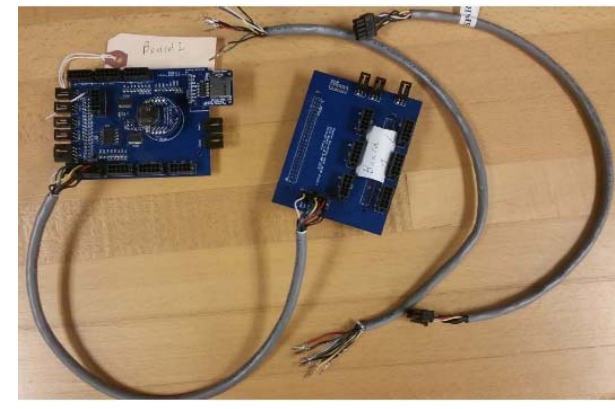

(b)

Figure 6. Implementation of the Microcontroller Module: (a) Breadboard Implementation (b) two PCBs, one for integration of the subsystems and the other as the breakout board 


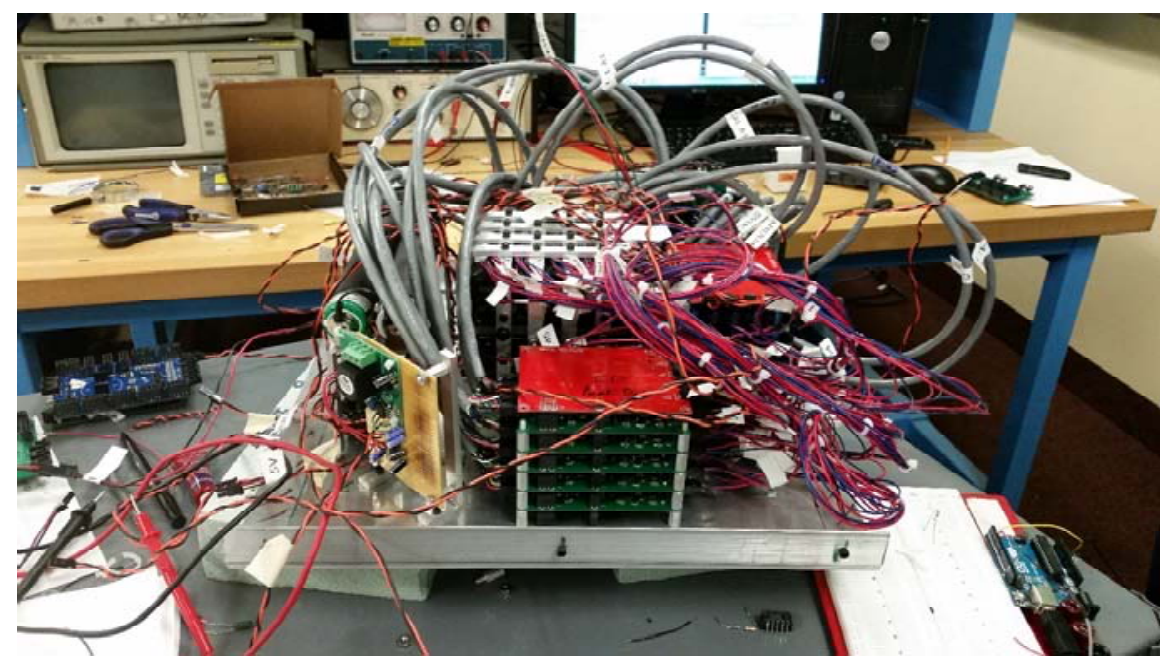

(a) Internal view of a previous cosmic-ray detector of similar dimension

Figure 7. Internal View of the previous payload

\section{Quantification of Project "Characteristics"}

\subsection{Primary Activities for a Project}

As described above, these two projects have different types of primary activities performed by students. To quantify the degree of "difference" in primary activities, we came up with a set of 15 qualitative statements, shown in Table 1. These questions, which may be subject to future refinement, are intended to represent typical engineering student-research activities in relation to assessing student learning outcomes in an engineering curriculum, particularly electrical engineering.

\subsection{Quantitative Measures}

To quantify the similarity or dissimilarity in primary activities of the two example projects, we adopt the Cohen's kappa [5] and apply quantitative interpretations of the strength of agreement [6] to quantitatively deducing similarity/dissimilarity from two sets of survey data on student's perception, based on the qualitative statements. The original Cohen's kappa coefficient is a statistic which measures inter-rater agreement for qualitative (categorical) items and is intended to measure the agreement between two raters who, respectively, classify $\mathrm{N}$ items into $\mathrm{C}$ mutually exclusive categories (for example, see [7]). In our application of Cohen's kappa, a rater evaluates two projects responding to a questionnaire of 15 qualitative statements to quantify the degree of similarity/dissimilarity (as opposed to the strength of agreement/disagreement) in primary project activities.

The value of Cohen's kappa is calculated by [7]

$$
\kappa=\frac{p_{o}-p_{e}}{1-p_{e}}
$$


Table 1. A questionnaire of 15 questions on project activities

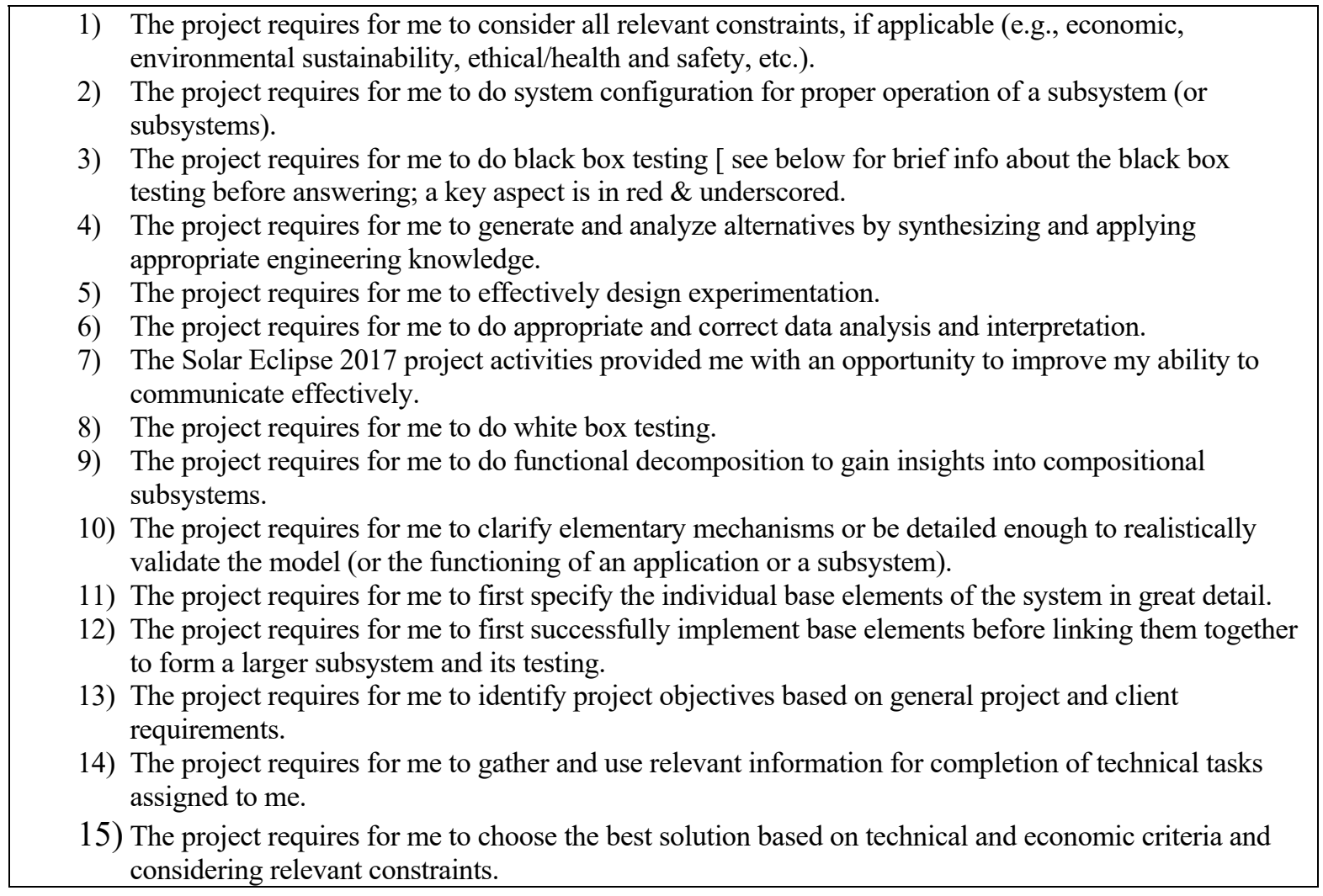

where $p_{o}$ is the relative number of observations in agreement and $p_{e}$ is the probability of an agreement occurring by chance.

\section{Survey Data and Quantitative Analysis}

For calculation of Cohen's kappa for each question, let us consider Q1 as an example. The survey question Q1 resulted in the following survey data on a scale of 1 through 5 from 7 students who have been involved in both projects for more than 2 semesters:

\begin{tabular}{|r|c|c|c|c|c|c|c|c|}
\hline Student $==>$ & $\# 1$ & $\# 2$ & $\# 3$ & $\# 4$ & $\# 5$ & $\# 6$ & $\# 7$ & av. \\
\hline Solar Eclipse & 4 & 3 & 5 & 5 & 4 & 3 & 4 & 4.00 \\
\hline CHERP & 3 & 3 & 5 & 5 & 4 & 3 & 4 & 3.86 \\
\hline
\end{tabular}

We construct a matrix of occurrences as follows:

\begin{tabular}{|l|l|l|l|l|l|l|}
\hline \multirow{2}{*}{ Q1 } & \multicolumn{6}{|l|}{ A: Solar Eclipse Ratings } \\
\cline { 3 - 7 } \multicolumn{2}{|c|}{5} & 5 & 4 & 3 & 2 & 1 \\
\hline \multirow{3}{*}{$\begin{array}{l}\text { B: } \\
\text { CHERP }\end{array}$} & 5 & $\mathbf{2}$ & 0 & 0 & 0 & 0 \\
\cline { 2 - 7 } & 4 & 0 & 2 & 0 & 0 & 0 \\
\cline { 2 - 7 } & 3 & 0 & 1 & 2 & 0 & 0 \\
\cline { 2 - 7 } & 2 & 0 & 0 & 0 & 0 & 0 \\
\cline { 2 - 7 } & 1 & 0 & 0 & 0 & 0 & 0 \\
\hline
\end{tabular}


where, for instance, the number ' 2 ' in the first cell (in bold face) represents 2 students rated both the solar eclipse project and CHERP project with a score of ' 5 ' on a scale of 1 through 5:

1-Strongly Disagree 2-Moderately Disagree 3- Neutral

4- Moderately Agree 5- Strongly Agree 6-Not Applicable

Although the response to all questions could have a score of 6 , no one responded with 6-Not Applicable. As such, the score range is presented on a scale of 1 through 5 only. From this table, the proportions of occurrences can be obtained by dividing each cell by the total number of responses, i.e., 7 , resulting in the following:

\begin{tabular}{|l|c|c|c|c|c|l|l|}
\hline \multirow{2}{*}{ Q1 } & \multicolumn{5}{|c|}{ A: Solar Eclipse Ratings } & \multirow{2}{*}{$p_{i, B}$} \\
\cline { 2 - 7 } & 5 & 4 & 3 & 2 & 1 & \\
\hline \multirow{3}{*}{$\begin{array}{l}\text { B: } \\
\text { CHERP } \\
\text { ratings }\end{array}$} & 5 & 0.2857 & 0 & 0 & 0 & 0 & 0.2857 \\
\cline { 2 - 7 } & 4 & 0 & 0.2857 & 0 & 0 & 0 & 0.2857 \\
\cline { 2 - 7 } & 2 & 0 & 0.1419 & 0.2857 & 0 & 0 & 0.4286 \\
\cline { 2 - 7 } & 1 & 0 & 0 & 0 & 0 & 0 & 0 \\
\hline \multirow{2}{*}{$p_{i, A}$} & 0.2857 & 0.4286 & 0.2857 & 0 & 0 & $\sum p_{i}=1$ \\
\hline
\end{tabular}

where the entities in the last row, $p_{i, A}$, is the sum of the proportions of the columns, and the entities in the last column, $p_{i, B}$, is the sum of the proportions of the rows. By adding the numbers in the main diagonal cells of the proportions matrix, we obtain $p_{o}=0.8571$. To find $p_{e}$, we first find joint probabilities of marginals by $p_{i}=p_{i, B} p_{i, A}$ and summarized them as follows:

\begin{tabular}{|c|c|c|c|c|c|c|}
\hline \multirow{2}{*}{\multicolumn{2}{|c|}{ Q1 }} & \multicolumn{5}{|c|}{ A: Solar Eclipse Ratings } \\
\hline & & 5 & 4 & 3 & 2 & 1 \\
\hline \multirow{5}{*}{$\begin{array}{l}\text { B: } \\
\text { CHERP } \\
\text { ratings }\end{array}$} & 5 & 0.0816 & 0.1224 & 0.0816 & 0 & 0 \\
\hline & 4 & 0.0816 & 0.1224 & 0.0816 & 0 & 0 \\
\hline & 3 & 0.1224 & 0.1837 & 0.1224 & 0 & 0 \\
\hline & 2 & 0 & 0 & 0 & 0 & 0 \\
\hline & 1 & 0 & 0 & 0 & 0 & 0 \\
\hline
\end{tabular}

Then, by adding the numbers in the main diagonal cells of the joint probabilities, we obtain the overall probability of random agreement $p_{e}=0.3265$. Finally, we obtain using (1), a Cohen's kappa value, $\kappa=0.7879$.

To calculate Cohen's kappa values for all questions, the entire survey results are given in Table 2 and the corresponding values of Cohen's kappa are calculated and summarized in Table 3.

Although there is no standard for interpreting the value of Cohen's kappa, the most widely cited interpretation [6] is that the best possible strength of agreement between raters is when $\kappa=1.0$, values in the range of $0<\kappa<1.0$ represent varying levels of agreement from slight to substantial, and $\kappa<0$ indicates poor agreement [7]. In our case, we consider that the larger a Cohen's kappa value is in the range of $0<\kappa<1.0$, the more similar the two projects are in inherent characteristics of offering the surveyed type of project activity. We then deduce that the higher the rate is, the 
Table 2. Survey results on project activities

\begin{tabular}{|c|c|c|c|c|c|c|c|c|c|c|c|c|c|c|c|c|c|}
\hline $\begin{array}{c}\text { Students } \\
=>\end{array}$ & $\# 1$ & $\# \mathbf{2}$ & $\mathbf{\# 3}$ & $\mathbf{\# 4}$ & $\mathbf{\# 3}$ & $\mathbf{\# 6}$ & $\mathbf{\# 7}$ & $\mathbf{a v}$ & & $\mathbf{1 1}$ & $\mathbf{\# 2}$ & $\mathbf{\# 3}$ & $\mathbf{\# 4}$ & $\mathbf{\# 5}$ & $\mathbf{\# 6}$ & $\# \mathbf{7}$ & $\mathbf{a v .}$ \\
\hline $\begin{array}{l}\text { Set A: } \\
\text { SE2017 }\end{array}$ & & & & & & & & & $\begin{array}{l}\text { Set B: } \\
\text { CHERP }\end{array}$ & & & & & & & & \\
\hline Q1 & 4 & 3 & 5 & 5 & 4 & 3 & 4 & 4.00 & Q1 & 3 & 3 & 5 & 5 & 4 & 3 & 4 & 3.86 \\
\hline Q2 & 4 & 5 & 4 & 5 & 4 & 5 & 5 & 4.57 & Q2 & 3 & 5 & 5 & 5 & 4 & 5 & 4 & 4.43 \\
\hline Q3 & 4 & 3 & 4 & 5 & 4 & 5 & 4 & 4.14 & Q3 & 3 & 4 & 5 & 5 & 4 & 5 & 5 & 4.43 \\
\hline Q4 & 4 & 5 & 5 & 5 & 4 & 5 & 5 & 4.71 & Q4 & 3 & 5 & 5 & 5 & 5 & 5 & 4 & 4.57 \\
\hline Q5 & 4 & 3 & 5 & 5 & 4 & 5 & 5 & 4.43 & Q5 & 2 & 5 & 5 & 5 & 5 & 5 & 5 & 4.57 \\
\hline Q6 & 3 & 4 & 5 & 5 & 4 & 5 & 5 & 4.43 & Q6 & 4 & 5 & 4 & 5 & 5 & 5 & 5 & 4.71 \\
\hline Q7 & 4 & 5 & 5 & 5 & 5 & 4 & 4 & 4.57 & Q7 & 3 & 4 & 4 & 5 & 4 & 5 & 4 & 4.14 \\
\hline Q8 & 1 & 2 & 4 & 5 & 4 & 3 & 4 & 3.29 & Q8 & 1 & 4 & 5 & 5 & 5 & 5 & 3 & 4.00 \\
\hline Q9 & 4 & 5 & 5 & 5 & 4 & 4 & 4 & 4.43 & Q9 & 3 & 5 & 5 & 4 & 4 & 5 & 4 & 4.29 \\
\hline Q10 & 4 & 5 & 4 & 5 & 4 & 5 & 5 & 4.57 & Q10 & 3 & 4 & 5 & 5 & 4 & 5 & 4 & 4.29 \\
\hline Q11 & 4 & 4 & 4 & 5 & 4 & 5 & 4 & 4.29 & Q11 & 2 & 3 & 5 & 4 & 4 & 5 & 5 & 4.00 \\
\hline Q12 & 4 & 4 & 5 & 5 & 4 & 5 & 5 & 4.57 & Q12 & 3 & 5 & 5 & 5 & 5 & 5 & 4 & 4.57 \\
\hline Q13 & 4 & 5 & 5 & 5 & 4 & 5 & 4 & 4.57 & Q13 & 3 & 3 & 4 & 5 & 5 & 5 & 4 & 4.14 \\
\hline Q14 & 4 & 5 & 5 & 5 & 4 & 5 & 4 & 4.57 & Q14 & 2 & 4 & 4 & 5 & 4 & 5 & 4 & 4.00 \\
\hline Q15 & 4 & 5 & 5 & 5 & 4 & 4 & 4 & 4.43 & Q15 & 3 & 4 & 5 & 5 & 4 & 4 & 4 & 4.14 \\
\hline
\end{tabular}

Table 3. Cohen's kappa values

\begin{tabular}{|c|c|c|c|}
\hline & SE average & CHERP average & Cohen's kappa \\
\hline Q1 & 4.00 & 3.86 & 0.7879 \\
\hline Q2 & 4.57 & 4.43 & 0.2222 \\
\hline Q3 & 4.14 & 4.43 & 0.1250 \\
\hline Q4 & 4.71 & 4.57 & 0.0455 \\
\hline Q5 & 4.43 & 4.57 & 0.1600 \\
\hline Q6 & 4.43 & 4.71 & -0.1200 \\
\hline Q7 & 4.57 & 4.14 & -0.2069 \\
\hline Q8 & 3.29 & 4.00 & -0.1351 \\
\hline Q9 & 4.43 & 4.29 & 0.2500 \\
\hline Q10 & 4.57 & 4.29 & 0.00 \\
\hline Q11 & 4.29 & 4.00 & -0.0606 \\
\hline Q12 & 4.57 & 4.57 & -0.0769 \\
\hline Q13 & 4.57 & 4.14 & 0.0968 \\
\hline Q14 & 4.57 & 4.00 & 0.2759 \\
\hline Q15 & 4.43 & 4.14 & 0.4815 \\
\hline
\end{tabular}

more interest and appreciation students have for the project on the particular project activity. By grouping questions based on the kappa values, we can rearrange survey results as follows:

\begin{tabular}{|c|c|c|c|}
\hline & SE average & CHERP average & Cohen's kappa \\
\hline Q1 & 4.00 & 3.86 & 0.7879 \\
\hline Q15 & 4.43 & 4.14 & 0.4815 \\
\hline
\end{tabular}




\begin{tabular}{|c|c|c|c|}
\hline Q14 & 4.57 & 4.00 & 0.2759 \\
\hline Q9 & 4.43 & 4.29 & 0.2500 \\
\hline Q2 & 4.57 & 4.43 & 0.2222 \\
\hline Q5 & 4.43 & 4.57 & 0.1600 \\
\hline Q3 & 4.14 & 4.43 & 0.1250 \\
\hline Q13 & 4.57 & 4.14 & 0.0968 \\
\hline Q4 & 4.71 & 4.57 & 0.0455 \\
\hline Q10 & 4.57 & 4.29 & 0.00 \\
\hline Q11 & 4.29 & 4.00 & -0.0606 \\
\hline Q12 & 4.57 & 4.57 & -0.0769 \\
\hline Q6 & 4.43 & 4.71 & -0.1200 \\
\hline Q8 & 3.29 & 4.00 & -0.1351 \\
\hline Q7 & 4.57 & 4.14 & -0.2069 \\
\hline
\end{tabular}

From this, we can see that, for Q1 as an example, while both projects are highly similar $(\kappa=$ 0.7879 ) in requiring the students to consider all relevant constraints, students more favorably rated the integration and configuration (solar eclipse) project for that project activity. Similarly, for Q15, while both projects are fairly similar $(\kappa=0.4815)$ in requiring students to choose the best solution based on technical and economic criteria and considering relevant constraints, the solar eclipse project was slightly highly rated for that aspect. In contrast, for Q7 as an example, while the two projects are quite dissimilar $(\kappa=-0.2069)$ in providing students with an opportunity to improve the ability to communicate effectively, students more favorably rated the solar eclipse project. For Q8, while the two projects are dissimilar $(\kappa=-0.1351)$ in requiring students to do white box testing, students rated higher for the design and implementation (CHERP) project.

Survey results for Student Outcomes - While both projects exhibit similarities and dissimilarities in certain project activities, we further assess how students rate each project in achieving student learning outcomes and the survey results are summarized below:

\begin{tabular}{|c|c|c|}
\hline Questions associated with ABET "a thru k" Student Outcomes & SE2017 & CHERP \\
\hline $\begin{array}{l}\text { (a) The Solar Eclipse } 2017 \text { (CHERP) project activities provided me with an opportunity to } \\
\text { improve my ability to apply knowledge of mathematics, science, and engineering. }\end{array}$ & 4.71 & 4.71 \\
\hline $\begin{array}{l}\text { a. Participating in the Solar Eclipse } 2017 \text { (CHERP) project activities, I have improved my } \\
\text { ability to apply knowledge of mathematics, science, and engineering. }\end{array}$ & 4.57 & 4.57 \\
\hline $\begin{array}{l}\text { (b) The Solar Eclipse } 2017 \text { (CHERP) project activities provided me with an opportunity to } \\
\text { improve my ability to design and conduct experiments, as well as to analyze and interpret } \\
\text { data. }\end{array}$ & 4.14 & 4.57 \\
\hline $\begin{array}{l}\text { b. Participating in the Solar Eclipse } 2017 \text { (CHERP) project activities, I have improved my } \\
\text { ability to design and conduct experiments, as well as to analyze and interpret data. }\end{array}$ & 4.14 & 4.71 \\
\hline $\begin{array}{l}\text { (c) The Solar Eclipse } 2017 \text { (CHERP) project activities provided me with an opportunity to } \\
\text { improve my ability to design a system, component, or process to meet desired needs within } \\
\text { realistic constraints. }\end{array}$ & 4.14 & 4.29 \\
\hline $\begin{array}{l}\text { c. Participating in the Solar Eclipse } 2017 \text { (CHERP) project activities, I have improved my } \\
\text { ability to design a system, component, or process to meet desired needs within realistic } \\
\text { constraints. }\end{array}$ & 4.43 & 4.14 \\
\hline $\begin{array}{l}\text { (d) The Solar Eclipse } 2017 \text { (CHERP) project activities provided me with an opportunity to } \\
\text { improve my ability to function on multidisciplinary teams. }\end{array}$ & 4.29 & 4.00 \\
\hline
\end{tabular}




\begin{tabular}{|c|c|c|}
\hline Questions associated with ABET "a thru k" Student Outcomes & SE2017 & CHERP \\
\hline $\begin{array}{l}\text { d. Participating in the Solar Eclipse } 2017 \text { (CHERP) project activities, I have improved my } \\
\text { ability to function on multidisciplinary teams. }\end{array}$ & 4.29 & 4.00 \\
\hline $\begin{array}{l}\text { (e) The Solar Eclipse } 2017 \text { (CHERP) project activities provided me with an opportunity to } \\
\text { improve my ability to identify, formulate, and solve engineering problems. }\end{array}$ & 4.43 & 4.43 \\
\hline $\begin{array}{l}\text { e. Participating in the Solar Eclipse } 2017 \text { (CHERP) project activities, I have improved my } \\
\text { ability to identify, formulate, and solve engineering problems. }\end{array}$ & 4.29 & 4.29 \\
\hline $\begin{array}{l}\text { (f) The Solar Eclipse } 2017 \text { (CHERP) project activities provided me with an opportunity to } \\
\text { improve my understanding of professional and ethical responsibility. }\end{array}$ & 3.86 & 3.86 \\
\hline $\begin{array}{l}\text { f. Participating in the Solar Eclipse } 2017 \text { (CHERP) project activities, I have improved my } \\
\text { understanding of professional and ethical responsibility. }\end{array}$ & 4.14 & 4.00 \\
\hline $\begin{array}{l}\text { (g) The Solar Eclipse } 2017 \text { (CHERP) project activities provided me with an opportunity to } \\
\text { improve my ability to communicate effectively. }\end{array}$ & 4.14 & 4.14 \\
\hline $\begin{array}{l}\text { g. Participating in the Solar Eclipse } 2017 \text { (CHERP) project activities, I have improved my } \\
\text { ability to communicate effectively. }\end{array}$ & 4.14 & 4.29 \\
\hline $\begin{array}{l}\text { (h) The Solar Eclipse } 2017 \text { (CHERP) project activities provided me with an opportunity to } \\
\text { broaden my education necessary to understand the impact of engineering solutions in a } \\
\text { global, economic, environmental, and societal context. }\end{array}$ & 3.86 & 3.71 \\
\hline $\begin{array}{l}\text { h. Participating in the Solar Eclipse } 2017 \text { (CHERP) project activities, I have broadened my } \\
\text { education necessary to understand the impact of engineering solutions in a global, } \\
\text { economic, environmental, and societal context. }\end{array}$ & 3.86 & 3.71 \\
\hline $\begin{array}{l}\text { (i) The Solar Eclipse } 2017 \text { (CHERP) project activities provided me with an opportunity to } \\
\text { improve my recognition of the need for, and an ability to engage in life-long learning. }\end{array}$ & 4.14 & 4.14 \\
\hline $\begin{array}{l}\text { i. Participating in the Solar Eclipse } 2017 \text { (CHERP) project activities, I have improved my } \\
\text { recognition of the need for, and an ability to engage in life-long learning. }\end{array}$ & 4.14 & 4.29 \\
\hline $\begin{array}{l}\text { (j) The Solar Eclipse } 2017 \text { (CHERP) project activities provided me with an opportunity to } \\
\text { enhance my knowledge of contemporary issues. }\end{array}$ & 4.00 & 3.29 \\
\hline $\begin{array}{l}\text { j. Participating in the Solar Eclipse } 2017 \text { (CHERP) project activities, I have enhanced my } \\
\text { knowledge of contemporary issues. }\end{array}$ & 3.71 & 3.43 \\
\hline $\begin{array}{l}\text { (k) The Solar Eclipse } 2017 \text { (CHERP) project activities provided me with an opportunity to } \\
\text { improve my ability to use the techniques, skills, and modern engineering tools necessary } \\
\text { for engineering practice. }\end{array}$ & 4.43 & 4.86 \\
\hline $\begin{array}{l}\text { k. Participating in the Solar Eclipse } 2017 \text { (CHERP) project activities, I have improved my } \\
\text { ability to use the techniques, skills, and modern engineering tools necessary for } \\
\text { engineering practice. }\end{array}$ & 4.29 & 4.86 \\
\hline
\end{tabular}

Although none of the student learning outcomes is intended to gauge effectiveness of a specific project or a single course, it still provides insights on how a specific project contributes to achieving specific student learning outcomes. From the results above, the design project (CHERP) appears to be more effective in achieving student outcomes (b) and (k) as expected, given the nature of the project activities of CHERP and as calculated values of Cohen's kappa by questions $3,5,6$, and 8 showing a relatively high degree of dissimilarities (i.e., perceived to be more leaning towards the design and implementation aspects). On the other hand, the results above also imply 
that either type of a project could contribute to effectively achieving the majority of the student learning outcomes.

\section{Concluding Remarks}

We have presented two exemplary projects each of which offers project activities leaning more toward either 1) integration and configuration or 2) design and implementation. Surveying a group

of undergraduate engineering students who have participated in both projects over a period of more than 2 semesters, we calculated and analyzed Cohen's kappa values to deduce similarities and dissimilarities from student perceptions on project activities. Our analysis indicated that the quantification technique using the Cohen's kappa could be a useful tool to evaluate similarities of two engineering projects in addressing student learning outcomes, provided that a set of qualitative statements is devised to accurately map them to key student learning outcomes for the intended characterization.

\section{Acknowledgement}

The projects described in this paper are supported in part by NASA Grant \#NNX16AL70A, and sub awards \#5373 \& \#5501-GU-NASA-K06H of NASA Grant \#NNX15AK06H.

\section{References}

[1] NASA's Strategic Management Council, NASA Education Strategic Coordination Framework: A Portfolio Approach, Feb., 2006. Available: http://www.hq.nasa.gov/office/hqlibrary/documents/o76805157.pdf.

[2] W. Lee and N. B. Conklin, "Improving student learning experience via extracurricular undergraduate research in near-space ballooning," FIE, Oct. 12-15, 2016, Erie, PA, pp. 1-5.

[3] Eclipse Ballooning Project: About the Eclipse Ballooning Project Systems, available at http://eclipse.montana.edu/about-the-systems/.

[4] W. Lee and N. B. Conklin, "Solar Eclipse Ballooning with a Multiband Tracking Subsystem for Undergraduate Research Experience,” ASEE, Jun. 25-28, 2017, Columbus, OH, pp. 1-12.

[5] J. Cohen, "A coefficient of agreement for nominal scales," Educational and Psychological Measurement, no. 20, pp. 37-46, 1960.

[6] J.R. Landis and G.G. Koch, "The measurement of observer agreement for categorical data," Biometrics, no. 33, pp. 159-74, 1977.

[7] S.J. Dickerson, S.P. Jacobs, A.M. Garcia and D.V.P. Sanchez, "Joint assessment and evaluation of senior design projects by faculty and industry, FIE 2016, Erie, PA, pp. 1-7. 